\title{
RISM-Assisted Analysis of the Role of Alkali Metal Hydroxides in the Solvation of Cellulose in Alkali/urea Aqueous Solutions
}

\section{Eugene Huh}

Yonsei University

Ji-Hyun Yang

Yonsei University

Chang-Ha Lee

Yonsei University

Ik-sung Ahn ( $\nabla$ iahn@yonsei.ac.kr)

Yonsei University https://orcid.org/0000-0002-1347-2037

Byung Jin Mhin

Paichai University: Pai Chai University

\section{Research Article}

Keywords: Cellulose, reference interaction site model (RISM), Kirkwood-Buff integral (KBI), alkali metal hydroxide, urea

Posted Date: May 10th, 2021

DOl: https://doi.org/10.21203/rs.3.rs-425463/v1

License: (c) (i) This work is licensed under a Creative Commons Attribution 4.0 International License. Read Full License

Version of Record: A version of this preprint was published at Cellulose on October 27th, 2021. See the published version at https://doi.org/10.1007/s10570-021-04214-w. 


\section{RISM-assisted analysis of the role of alkali \\ 2 metal hydroxides in the solvation of cellulose \\ 3 in alkali/urea aqueous solutions}

4 Eugene Huh ${ }^{\dagger}$, Ji-Hyun Yang ${ }^{\dagger}$, Chang-Ha Lee ${ }^{\dagger}$, Ik-Sung Ahn ${ }^{*}{ }^{\dagger}$, Byung Jin Mhin ${ }^{*},+$

$5{ }^{t}$ Department of Chemical and Biomolecular Engineering, Yonsei University,

6 Seoul 120-749, South Korea

$7 \quad{ }^{\ddagger}$ Department of Chemistry, Paichai University, Daejeon 302-735, South Korea

8

$9 *$ Corresponding authors

10 Tel.: +(82) 2-2123-2752, +(82) 42-520-5611

11

12 Eugene Huh: eugeneh55@gmail.com

13 Ji-Hyun Yang: prji17@naver.com

14 Chang-Ha Lee: leech@yonsei.ac.kr ORCID 0000-0001-8769-7936

15 Ik-Sung Ahn*: iahn@yonsei.ac.kr ORCID 0000-0002-1347-2037

16 Byung Jin Mhin*: mhin@pcu.ac.kr

18 The three-dimensional reference interaction site model theory with the Kovalenko-Hirata closure

19 (3D-RISM-KH) combined with the Kirkwood-Buff integral (KBI) was used to clarify the role of

20 alkali metal hydroxides (MOHs) in cellulose solvation in alkali/urea aqueous solutions. The KBI

21 and excess number of MOHs showed that their access and affinity to cellulose were both in the

22 order $\mathrm{LiOH}>\mathrm{NaOH}>\mathrm{KOH}$. The cavity and interaction volumes for cellulose indicate that the

23 alkali metal ions closer in proximity to cellulose induce thermal fluctuations of cellulose molecules

24 more easily and cause more electrostatic interactions and hydrogen bonding with cellulose. These

25 calculation results support the hypothesis of cellulose charging up, which claims that cellulose-ion

26 interactions cause cellulose solvation. The energy and chemical potential of cellulose solvation

27 confirmed the affinity and interaction of MOHs with cellulose. Solvent reorganization, whose

28 energy primarily determined the solvation energy, was found to be facilitated by the more

29 proximal MOH when in the presence of urea. 3D-RISM-KH combined with KBI produced

30 quantitative information regarding the distribution and attractive interaction of MOHs (especially

$31 \mathrm{LiOH}$ ) with cellulose, which clarified their role in cellulose solvation in alkali/urea aqueous

32 solutions.

34 Keywords: Cellulose, reference interaction site model (RISM), Kirkwood-Buff

35 integral (KBI), alkali metal hydroxide, urea 


\section{Introduction}

38 Recently, aqueous solutions of urea and various kinds of alkali metal hydroxides

39 (e.g., $\mathrm{LiOH}, \mathrm{NaOH}$, and $\mathrm{KOH}$ ) have shown different solvation capabilities toward

40 cellulose molecules of varying molecular weights (Cai and Zhang 2005; Wang et

41 al. 2017). Three cellulose molecules with viscosity-average molecular weights of

$424.5 \times 104,11.5 \times 104$ and $37.2 \times 104$ were found to be soluble in a $4.2 \%(\mathrm{w} / \mathrm{w})$

$43 \mathrm{LiOH} / 12 \%(\mathrm{w} / \mathrm{w})$ urea solution precooled to $-10^{\circ} \mathrm{C}$ (Cai and Zhang 2005). A $7 \%$

44 (w/w) $\mathrm{NaOH} / 12 \%(\mathrm{w} / \mathrm{w})$ urea solution could dissolve the first two cellulose

45 molecules, while a $9.8 \%(\mathrm{w} / \mathrm{w}) \mathrm{KOH} / 12 \%(\mathrm{w} / \mathrm{w})$ urea solution did not dissolve

46 any of the tested cellulose molecules. Using differential scanning calorimetry

47 analysis, Wang et al. (Wang et al. 2017) estimated cellulose solvation to be

48 feasible at approximately $-5^{\circ} \mathrm{C}$ in $\mathrm{LiOH} / \mathrm{urea}$ solutions but at $-20^{\circ} \mathrm{C}$ in $\mathrm{NaOH} / \mathrm{urea}$

49 solutions. By measuring pulsed field-gradient spin-echo NMR intensities, they

50 calculated that the ratios of $\mathrm{Li}^{+}$and $\mathrm{Na}^{+}$bound to cellulose were $14.3 \%$ and 4.8

$51 \%$, respectively. These experimental results indicate that these ions' interaction

52 with cellulose and contribution to cellulose solvation are in the order $\mathrm{Li}^{+}>\mathrm{Na}^{+}>$

$53 \mathrm{~K}^{+}$. The results of a molecular dynamics (MD) simulation suggest that the

54 interaction between cellulose and alkali metal hydroxides such as $\mathrm{LiOH}$ and

$55 \mathrm{NaOH}$ is primarily an electrostatic interaction. In their calculation, $\mathrm{Li}^{+}$ions were

56 closer in proximity to cellulose than $\mathrm{Na}^{+}$ions, and the average distance of $\mathrm{Li}^{+}$

57 from the oxygens of cellulose was smaller than that of $\mathrm{Na}^{+}$. The probability of

58 finding $\mathrm{Li}^{+}$ions within the same distance of cellulose was higher than that of

59 finding $\mathrm{Na}^{+}$ions (Wang et al. 2017). Xiong et al. (Xiong et al. 2013) investigated

60 the dissolution of cellobiose, a unit of cellulose, in alkali solutions to clarify the

61 role of alkali metal ions in cellulose solvation. In the ${ }^{13} \mathrm{C}-\mathrm{NMR}$ spectroscopy of

62 cellobiose, a larger downfield chemical shift and shorter ${ }^{13} \mathrm{C}$ relaxation time were

63 detected in the order $\mathrm{LiOH}>\mathrm{NaOH}>\mathrm{KOH}$. This means that the interaction

64 between cellobiose and alkali metal ions is on the same order, which is consistent

65 with the contribution of alkali metal ions to cellulose solvation (Xiong et al.

66 2013).

67 In our previous work (Huh et al. 2020), the three-dimensional reference

68 interaction site model theory with the Kovalenko-Hirata closure (3D-RISM-KH)

69 was applied to calculate the solvation energy of cellulose in $\mathrm{NaOH} /$ urea aqueous

70 solution at $261 \mathrm{~K}$ and to determine the contribution of each solvent species (i.e., 
$71 \mathrm{Na}^{+}, \mathrm{OH}^{-}$, urea, and water) to cellulose solvation. Water molecules were found to

72 be located nearer to cellulose in $\mathrm{NaOH} /$ urea aqueous solutions than in $\mathrm{NaOH}$

73 solutions without urea. As a result, the inclusion of urea caused a reduction in the

74 solvent reorganization energy of cellulose from 163 to $-1,198 \mathrm{kcal} / \mathrm{mole}$ and a

75 decrease in the interaction energy between cellulose and the solvent by more than

$76100 \mathrm{kcal} / \mathrm{mole}$.

77 In this work, a system for cellulose solvation was extended to aqueous solutions

78 of urea and various alkali metal hydroxides, such as $\mathrm{LiOH}, \mathrm{NaOH}$, and $\mathrm{KOH}$. The

79 objective of this work is to determine the role of alkali metal hydroxides in

80 cellulose solvation (i.e., their affinity to cellulose) by calculating and comparing

81 the distribution of the solvent species and the partial molar volume and solvation

82 energy of cellulose. For this purpose, 3D-RISM-KH (Gusarov et al. 2012;

83 Kovalenko 2013, 2017; Kovalenko and Gusarov 2018) combined with the

84 Kirkwood-Buff integral (KBI) (Giambaşu et al. 2015; Giambaşu et al. 2014;

85 Krüger et al. 2013) was used in this study.

\section{Methods}

88 The KBI, $G_{\gamma}$ is defined as the spatial integral over the pair distribution function $89 \quad\left(g_{\gamma}^{u v}(r)\right)$ :

$$
G_{\gamma}=\int_{V}\left[g_{\gamma}^{u v}(r)-1\right] d r
$$

91 where superscripts $\mathrm{u}$ and $\mathrm{v}$ denote the solute and solvent, respectively; $\mathrm{V}$ is the

92 system volume over which the integration is carried out; and $\mathrm{r}$ is the distance

93 between the solute and the solvent species. $g_{\gamma}^{u v}(r)$ is the ratio of the probability

94 of finding the solvent species $\gamma$ at $\mathrm{r}$, which is determined from the 3D-RISM

95 calculations, to the probability of finding $\gamma$ in the bulk solvent phase. The unit of

$96 G_{\gamma}$ is the volume per single solute molecule $\left(\AA^{3} /\right.$ molecule $) . G_{\gamma}$ indicates the 97 accessibility of $\gamma$ to the solute, which is correlated with the affinity of $\gamma$ to the 98 solute (Nicol et al. 2017; Wernersson et al. 2015). The KBI has been considered 99 appropriate for describing all types of intermolecular interactions necessary for

100 determining the thermodynamic properties of a solution. For example, it has been

101 used to calculate the excess number of $\gamma$ around the single solute molecule,

$102 N_{\gamma}^{\text {excess }}$ as follows (Shimizu 2004; Shimizu et al. 2013): 


$$
N_{\gamma}^{\text {excess }}=\rho_{\gamma}\left(G_{\gamma}+V_{C}\right)
$$

104 where $\rho_{\gamma}$ is the number density of $\gamma$ and $V_{C}$ is the excluded volume of the

105 solute. The sum of $G_{\gamma}$ and $V_{C}$ corresponds to the real volume in which $\gamma$ can

106 exist near the solute. The excess numbers of various solvent species allow us to

107 compare their interaction with the solute.

108 The partial molar volume of the solute, $\bar{V}$, is also affected by its interaction with

109 the solvent species and is defined as follows (Chalikian and Breslauer 1996; Imai

110 2007a; Imai et al. 2007b; Patel et al. 2011):

$$
\bar{V}=V_{C}+V_{I}+V_{i d}
$$

112 where $V_{I}$ and $V_{i d}$ denote the interaction volume and the ideal fluctuation

113 volume, respectively. $V_{i d}$ is caused by the translational degrees of freedom of the

114 solvent (Imai 2007a). $V_{C}$, which is also called the cavity volume, consists of the

115 region occupied by the solute $\left(V_{M}\right.$, the geometric volume) and an empty border

116 region between the solute and the unaltered, uniform pure solvent $\left(V_{T}\right.$, the thermal

117 volume) (Lee 1983; Patel et al. 2011). $V_{T}$ results from thermally induced

118 molecular fluctuations between the solute and solvent species (Chalikian and

119 Breslauer 1996; Imai 2007a) and may simply be considered the void volume due

120 to their imperfect packing (Imai 2007a). $V_{I}$ represents the change in the solvent

121 volume due to the intermolecular electrostatic interaction and hydrogen bonding

122 between cellulose and solvent species (Chalikian and Breslauer 1996). In this

123 study, the more nonpolar and polar interaction of cellulose with the solvent will

124 cause smaller $V_{T}$ (i.e., $V_{C}$ ) and $V_{I}$, respectively, which will lead to a smaller $\bar{V}$.

125 The solvation energy $(\Delta E)$ and solvation entropy $(T \Delta S)$, which are needed for

126 calculating the excess chemical potential $(\Delta \mu)$, are defined as the sum of solute-

127 solvent interaction (uv) and solvent-solvent reorganization (R) terms:

$$
\Delta E=E^{u v}+\Delta E^{R}
$$

$$
T \Delta S=T \Delta S^{u v}+T \Delta S^{R}=T \Delta S^{u v}+\Delta E^{R}
$$

131 upon insertion of the solute molecule (Ben-Naim 1978; Gallicchio et al. 2000;

132 Lazaridis 2000; Misin 2017), they have the same values. Then

$$
\Delta \mu=\Delta E-T \Delta S=E^{u v}-T \Delta S^{u v}
$$

134 3D-RISM is known to strongly overestimate the excess chemical potential (Misin

135 2017; Sergiievskyi et al. 2014). Sergiievskyi et al. (Sergiievskyi et al. 2014)

136 showed that this is primarily caused by a high solvent pressure, which leads to 
137 overestimation of the solute insertion work. Misin (Misin 2017) argued that water

138 molecules near a large solute, such as cellulose, undergo substantial

139 reorganization. The density of the water molecules immediately next to the solute

140 is lower than that of bulk water molecules, which is caused by the lack of

141 attractive interaction with the hydrophobic moiety of the solute. Instead, they are

142 strongly drawn in by the bulk water, forming a strong tetrahedral network. These

143 solvent reorganizations create large cavities near the hydrophobic moiety of the

144 solute. Such a dewetting transition cannot be considered by conventional 3D-

145 RISM and requires pressure correction of 3D-RISM (Misin 2017). $\Delta \mu_{P C+}$, or $\Delta \mu$

146 corrected by including the advanced pressure correction (PC+) and the partial

147 molar volume of cellulose, is expressed as follows:

$148 \Delta \mu_{P C+}=\Delta \mu+\left(P_{i d}-P\right) \bar{V}$

149 where $P_{i d}$ is an ideal gas pressure of the solvent given by $P_{i d}=\rho_{T o t} k_{B} T$ and $P$

150 is given by $P=\frac{1}{2} k_{B} T\left(\frac{1}{k_{B} T \chi_{T}}+N_{\text {site }} \rho_{T o t}\right) \cdot \rho_{T o t}$ and $N_{\text {site }}$ are the number

151 densities of all solvent species and the number of sites (atoms) in the solvent,

152 respectively (Misin et al. 2016; Sergiievskyi et al. 2014).

153 The 3D-RISM-KH calculation and MD simulation were performed with the

154 AMBER16 package (Case et al. 2010). As a model cellulose molecule, the

155 molecule with the I $\beta$ structure and degree of polymerization of 8 was selected in

156 this study. The following eight solvents were tested for cellulose solvation at 261

$157 \mathrm{~K}$ : LiOH/urea aqueous solution (abbreviated as solvent LU), $\mathrm{NaOH} / \mathrm{urea}$ (solvent

$158 \mathrm{NU}$ ), $\mathrm{KOH} / \mathrm{urea}$ (solvent KU), pure water (solvent WO), urea aqueous solution

159 (solvent $\mathrm{UO}$ ), $\mathrm{LiOH}$ solution (solvent $\mathrm{LO}$ ), $\mathrm{NaOH}$ solution (solvent $\mathrm{NO}$ ), and

$160 \mathrm{KOH}$ solution (solvent $\mathrm{KO}$ ). The latter 5 solvents were used for comparison with

161 the first 3 solvents (LU, NU, and KU). For meaningful comparison of the

162 cellulose solvation thermodynamics and solvent affinities toward cellulose, the

163 number ratio of water, $\mathrm{MOH}$, and urea was maintained at 27.75:1:1. The 3D-

164 RISM-KH calculations were performed in a $32 \times 40 \times 72 \AA^{3}$ box with a spacing of

$1650.5 \AA$. TIP3P water was used as the solvent water. The detailed calculation

166 method is described in Supplementary Information. The initial density and

167 pressure values of each solvent are shown in Table S1 (see Supplementary

168 Information). The force-field parameters and partial charge were assigned using

169 the General Amber Force Field and AM1-BCC charge model, respectively

170 (Jakalian et al. 2000; Jakalian et al. 2002). The structure of the model cellulose 
171 molecule was created using a cellulose-builder (Gomes and Skaf 2012). The

172 geometric volume $\left(V_{M}\right)$ of cellulose was calculated using Protein Volume 1.3

173 (Chen and Makhatadze 2015).

\section{Results and discussion}

\section{Affinity of $\mathrm{Li}^{+}, \mathrm{Na}^{+}$, and $\mathrm{K}^{+}$toward cellulose}

177 Fig. 1 shows an example of the pair distribution functions used in Eq. (1). KBI

$178\left(G_{\gamma}\right)$ values were calculated for all solvent species in the 8 different solvents and

179 are shown in Fig. 2. The negative $G_{\gamma}$ values arise due to the excluded volume

$180\left(V_{C}\right)$ of cellulose (Sergiievskyi et al. 2014), and solvent species with a more

181 negative $G_{\gamma}$ have a lower probability of existing near cellulose and are less

182 accessible to cellulose. The $G_{\gamma}$ was found to be in the order urea $>\mathrm{LiOH}>$

183 Water $>\mathrm{NaOH}>\mathrm{KOH}$. $G_{\text {urea }}$ seems to be constant regardless of the other

184 solvent species, such as MOHs, which corresponds well with the positioning of

185 urea above and below the pyranose rings of cellulose and its interaction with

186 cellulose independent of MOH (Huh et al. 2020; Wernersson et al. 2015; Xiong et

187 al. 2013). $G_{M O H}$ was found to be in the order $\mathrm{LiOH}>\mathrm{NaOH}>\mathrm{KOH}$ regardless

188 of whether urea was present in the solvent. $G_{L i O H}$ was higher than $G_{\text {water }}$ in

189 solvents LO and LU, while $G_{N a O H}$ and $G_{K O H}$ were lower than $G_{\text {water }} \cdot \mathrm{Li}^{+}, \mathrm{Na}^{+}$,

190 and $\mathrm{K}^{+}$have the same charge but different sizes, which were in the order $\mathrm{Li}^{+}<$

$191 \mathrm{Na}^{+}<\mathrm{K}^{+}$(see Table S2 in Supplementary Information). Hence, $\mathrm{Li}^{+}$is most easily

192 accessible to cellulose, which enables the strongest electrostatic interaction and

193 corresponds well with the experimental finding that cellulose is most favorably

194 soluble in solvent LU. Due to these differences in the electrostatic interaction of

195 MOHs with cellulose, $G_{\text {water }}$ in the solvent containing $\mathrm{LiOH}$ was the smallest,

196 while that in the solvent containing $\mathrm{KOH}$ was the largest regardless of the

197 presence of urea (see Fig. 1).

198 Table 1 summarizes the $N_{\gamma}^{\text {excess }}$ of each solvent species in the 4 different

199 solvents. The $N_{\gamma}^{\text {excess }}$ values for cellulose in solvents WO, LO, NO, and KO are

200 shown in Table S4. As expected from $G_{M O H}$, the smaller alkali metal ion yielded

201 the larger $N_{M O H}^{\text {excess }}$ (i.e., $N_{L i O H}^{\text {excess }}>N_{N a O H}^{\text {excess }}>N_{K O H}^{\text {excess }}$ ). The greater

202 concentration close to cellulose implies a greater interaction between the

203 corresponding metal ion and cellulose and a higher probability of making 
204 cellulose into a polyelectrolyte in the order $\mathrm{Li}^{+}>\mathrm{Na}^{+}>\mathrm{K}^{+}$. Hence, the $N_{M O H}^{\text {excess }}$

205 data support the hypothesis of cellulose charging up (Nicol et al. 2017), which

206 claims that cellulose-ion interactions can make cellulose into a polyelectrolyte and

207 cause its solvation (Bialik et al. 2016).

208 As urea is positioned above and below the pyranose rings of cellulose, the region

209 near cellulose that is available for water and MOHs decreases upon the addition of

210 urea into the solvent. Hence, the difference between $N_{M O H}^{\text {excess }}$ and $N_{\text {water }}^{\text {excess }}$ is

211 smaller when urea is present in the solvent. Due to the interaction with cellulose

212 and the size of ion hydrates (see Table S2 in Supplementary Information), which

213 is in the order $\mathrm{Li}^{+}>\mathrm{Na}^{+}>\mathrm{K}^{+}, N_{\text {water }}^{\text {excess }}$ was dependent on the kind of $\mathrm{MOH}$ :

$214 N_{\text {water }}^{\text {excess }}$ in solvent with $\mathrm{LiOH}<N_{\text {water }}^{\text {excess }}$ in solvent with $\mathrm{NaOH}<N_{\text {water }}^{\text {excess }}$ in

215 solvent with $\mathrm{KOH}$ (see Table 1 and Fig. 3). Based on the $G_{\gamma}$ and $N_{\gamma}^{\text {excess }}$,

216 positioning of the solvent species around the single cellulose molecule is

217 schematically depicted in Fig. 3. Note that MOHs and urea are capable of

218 hydrophilic and hydrophobic interactions with cellulose, respectively (Huh et al.

$2192020)$.

221 Partial molar volume of cellulose dependent on alkali metal hydroxides

222 Cavity volume $\left(V_{C}\right)$ and its component for each solvent species $\gamma\left(V_{C, \gamma}\right)$ was

223 calculated for cellulose in solvents LU, NU, and KU and is shown in Tables 2 and

2243 , respectively. The geometric volume $\left(V_{M}\right)$, which is equal to the sum of the van

225 der Waals volume $\left(V_{W}\right)$ and void volume $\left(V_{V}\right)$, was calculated to be $1173.3 \AA^{3}$,

226 while $V_{W}$ and $V_{V}$ were $1061.7 \AA^{3}$ and $111.6 \AA^{3}$, respectively. Since $V_{M}$ is

227 constant regardless of the solvent, the difference in $V_{C}$ for different solvents

228 originates from the thermal volume $\left(V_{T}\right)$. To further study the dependence of $V_{C}$

229 on the kind of $\mathrm{MOH}, V_{C}$ was divided into three components: $V_{C, \text { water }}, V_{C, M O H}$,

230 and $V_{C, \text { urea }}$. It was found in a previous study (Huh et al. 2020) that urea was

231 predominantly positioned close to the hydrophobic part of cellulose (i.e., above

232 and below the pyranose rings of cellulose), while $\mathrm{MOH}$ was positioned close to

233 the hydrophilic part of cellulose (i.e., along the edges of cellulose). Hence,

$234 V_{C, \text { urea }}$ was not affected by MOHs (see Table 3).

235 The radial distribution functions of $\mathrm{Li}^{+}, \mathrm{Na}^{+}$, and $\mathrm{K}^{+}$shown in Fig. 1 indicate the

236 denser and more proximal distribution of $\mathrm{Li}^{+}$to cellulose than $\mathrm{Na}^{+}$and $\mathrm{K}^{+}$. Hence,

$237 G_{M O H}$ and $N_{M O H}^{\text {excess }}$ were in the order $\mathrm{LiOH}>\mathrm{NaOH}>\mathrm{KOH}$. MOHs smaller and 
nearer to cellulose are apt to more easily induce thermal fluctuation of cellulose

239 molecules and to result in the further reduction of $V_{C}$, which leads to $V_{C, M O H}$ in

240 the following order: $V_{C, \mathrm{LiOH}}<V_{C, \mathrm{NaOH}}<V_{C, \mathrm{KOH}}$. The magnitudes of $G_{\text {water }}$

241 and $N_{\text {water }}^{\text {excess }}$ are in the order solvent $\mathrm{LU}<$ solvent $\mathrm{NU}<$ solvent $\mathrm{KU}$; therefore,

242 we see the opposite order for $V_{C, \text { water }}: V_{C, \text { water }}$ in solvent LU $>V_{C, \text { water }}$ in

243 solvent $\mathrm{NU}>V_{C, \text { water }}$ in solvent KU. The $V_{C}$ values for cellulose in solvents

$244 \mathrm{LO}, \mathrm{NO}$, and $\mathrm{KO}$ are shown in Table S5, while their water and $\mathrm{MOH}$ components

245 are shown in Table S6. For the same $\mathrm{MOH}, V_{C, \text { water }}$ is smaller in the presence of

246 urea, which is consistent with the results of a previous study which indicated that

247 water molecules were positioned closer in proximity to cellulose in the presence

248 of urea (Huh et al. 2020).

249 Interaction volume $\left(V_{I}\right)$ and its component for each solvent species $\gamma\left(V_{I, \gamma}\right)$ was

250 calculated for cellulose in solvents LU, NU, and KU and is shown in Tables 2 and

2513 , respectively. $V_{I}$ was found to be in the order solvent LU (most negative) <

252 solvent NU < solvent KU (least negative); these results imply that the most

253 closely distributed LiOH (see Fig. 1) results in the most hydrophilic interaction

254 with cellulose. When separately calculating $V_{I, \text { water }}, V_{I, M O H}$, and $V_{I, \text { urea }}$, the

255 difference in $V_{I}$ between the three solvents could not be explained with $V_{I, M O H}$.

256 Even though $G_{M O H}$ and $N_{M O H}^{\text {excess }}$ were in the order $\mathrm{LiOH}>\mathrm{NaOH}>\mathrm{KOH}$, the

257 most negative $V_{I, M O H}$ caused by the most hydrophilic interaction between MOHs

258 and cellulose was found in solvent KU. The most negative $V_{I, \text { water }}$ was found in

259 solvent LU despite $G_{\text {water }}$ and $N_{\text {water }}^{\text {excess }}$ being in the order $\mathrm{KOH}>\mathrm{NaOH}>$

$260 \mathrm{LiOH}$. Therefore, MOHs might not be directly involved in the hydrophilic

261 interaction with cellulose. Instead, a portion of the water molecules were

262 positioned close to cellulose; for example, water molecules comprising ion

263 hydrates might predominantly participate in electrostatic interactions and

264 hydrogen bonding with cellulose, which explains the results for $V_{I, \text { water }}$ and

$265 V_{I, M O H}$ in Table 3 . The ideal fluctuation volume $\left(V_{i d}\right)$ was negligibly small

266 compared to $V_{C}$ and $V_{I}$ (see Table 2), and its influence on $\bar{V}$ did not need to be

267 considered. The $V_{I}$ values for cellulose in solvents $\mathrm{LO}, \mathrm{NO}$, and $\mathrm{KO}$ are shown

268 in Table S5, while their water and MOH components are shown in Table S6.

269 Significant differences are not found in $V_{C}$ and $V_{I}$, which implies that urea is

270 indispensable for facilitating MOH-thermally-induced molecular fluctuations of 
271 cellulose as well as causing water molecules in ion hydrates to participate in

272 electrostatic interaction and hydrogen bonding with cellulose.

274 Thermodynamics of cellulose solvation depending on alkali metal hydroxides

275 The solvation energy and entropy of cellulose in solvents LU, NU, and KU are

276 shown in Table 4, while those in solvents LO, NO, and KO are shown in Table

277 S7. The superscripts uv and R denote that the corresponding energy terms

278 consider solute-solvent interaction and solvent reorganization (i.e., solvent-solvent

279 interaction), respectively. The solvation energy accounted for the experimental

280 result of cellulose solvation (i.e., cellulose is most easily dissolved in solvent LU

281 and not soluble in solvent KU).

282 The $E^{u v}$ (direct solvation energy) in solvent LU is $-481.4 \mathrm{kcal} / \mathrm{mol}$ and smaller

283 than -470.9 and $-450.7 \mathrm{kcal} / \mathrm{mol}$ in solvents $\mathrm{NU}$ and $\mathrm{KU}$, respectively, which

284 indicates that $\mathrm{Li}^{+}$is positioned closest to cellulose and experiences the strongest

285 interaction with cellulose. Note that the difference in $E^{u v}$ was affected more

286 dominantly by MOHs than by water (compare the numbers in square and round

287 brackets on the column for $E^{u v}$ in Table 4). The following $\Delta E_{M O H-W O}^{u v}$ and

$288 \Delta E_{M O H-U O}^{u v}$ were calculated to determine the effect of urea on the contribution of

289 MOHs to $E^{u v}$ (see Table 5):

$$
\Delta E_{M O H-W O}^{u v}=E^{u v}(\text { solvent } L O, N O, K O)-E^{u v}(\text { solvent } W O)
$$

$$
\Delta E_{M O H-U O}^{u v}=E^{u v}(\text { solvent } L U, N U, K U)-E^{u v}(\text { solvent } U O)
$$

294 their difference is largest when the $\mathrm{MOH}$ is $\mathrm{LiOH}$. Urea is believed to strengthen

295 the interaction between cellulose and MOHs, especially $\mathrm{LiOH}$. The $T \Delta S^{u v}$ in

296 solvent LU is $-518.7 \mathrm{kcal} / \mathrm{mol}$ and higher (i.e., less negative) than those in the

297 other solvents, which indicates that the access of $\mathrm{LiOH}$ to cellulose is less

298 hindered than the access of $\mathrm{NaOH}$ and $\mathrm{KOH}$ to cellulose.

299 Table 4 separately shows the contributions of water and MOHs to $\Delta E^{R}$ (see the

300 numbers in round and square brackets on the column for $\left.\Delta E^{R}\right)$. The

301 reorganization of water and $\mathrm{MOHs}$ around cellulose is favored by type of $\mathrm{MOH}$ in

302 the order $\mathrm{LiOH}>\mathrm{NaOH}>\mathrm{KOH}$. The following $\Delta \Delta E_{M O H-W O}^{R}$ and $\Delta \Delta E_{M O H-U O}^{R}$

303 were calculated to determine the effect of urea on the contribution of MOHs to

$304 \Delta E^{R}$ (see Table 5): 
$307 \Delta E^{R}$ in the solvent containing urea is much smaller than that in the solvent

308 without urea (compare Tables 4 and S6). Note that $\Delta E^{R}$ decreased by

309 approximately $1300 \mathrm{kcal} / \mathrm{mol}$ in the presence of urea. As a result, $\Delta \Delta E_{M O H-U O}^{R}$ is

310 much more negative than $\Delta \Delta E_{M O H-W O}^{R}$, and their difference is largest when MOH

311 is $\mathrm{LiOH}$. Hence, it is believed that urea induces the reorganization of water and

$312 \mathrm{MOHs}$, especially $\mathrm{LiOH}$ around cellulose, to favor solvent-solvent interactions.

313 The chemical potentials of cellulose solvation were corrected by including

314 advanced pressure correction $(\mathrm{PC}+$ ) and are shown in Table 6 (see the column

315 marked with $\Delta \mu_{P C+}$. The $\Delta \mu_{P C+}$ values are $-193.8,-188.6$, and $-178.4 \mathrm{kcal} / \mathrm{mol}$

316 in solvents LU, NU, and KU, respectively, which agrees with the experimental

317 result of cellulose solvation in these solvents. $\Delta \mu_{P C+}$ in the solvent without urea

318 is shown in Table S8 and is higher (i.e., less negative) than that in the

319 corresponding solvent with urea (e.g., -139.8 and $-193.8 \mathrm{kcal} / \mathrm{mol}$ in solvents LO

320 and LU, respectively). Note that the difference in $\Delta \mu_{P C+}$ between MOHs is not

321 significant in the solvents without urea. The calculation results of $E^{u v}, \Delta E^{R}$, and

$322 \Delta \mu_{P C+}$ indicate that cellulose solvation in alkali/urea aqueous solutions was

323 feasible due to the presence of urea in the solvent, and the contribution of MOHs

324 was in the order $\mathrm{LiOH}>\mathrm{NaOH}>\mathrm{KOH}$.

\section{Conclusions}

326 Alkali metal hydroxides (MOHs) in aqueous urea solutions were reported to 327 favor cellulose solvation in the order $\mathrm{LiOH}>\mathrm{NaOH}>\mathrm{KOH}$, as determined from 328 the molecular weights of soluble cellulose and solvation temperatures. The three329 dimensional reference interaction site model theory with the Kovalenko-Hirata 330 closure (3D-RISM-KH) combined with the Kirkwood-Buff integral (KBI) was 331 used in this study to determine the role of MOHs in cellulose solvation. For this

332 purpose, the access and interaction of MOHs with cellulose was estimated by 333 calculating the KBI and excess number of solvent species around cellulose, the 334 partial molar volume of cellulose, and the thermodynamics of cellulose solvation.

335 The KBI and excess number of $\mathrm{MOH}$ sere in the order $\mathrm{LiOH}>\mathrm{NaOH}>\mathrm{KOH}$, 336 which indicates the positioning of $\mathrm{LiOH}$ (i.e., $\mathrm{Li}^{+}$) closest to cellulose and implies 337 the strongest electrostatic interaction. The KBI of urea was much higher than that 
338 of water, which agrees with the finding that urea prefers to be positioned near the

339 hydrophobic surface of cellulose and is involved in hydrophobic interactions with

340 cellulose independent of MOHs. The excess numbers of MOHs on the order of

$341 N_{\text {LiOH }}^{\text {excess }}>N_{N a O H}^{\text {excess }}>N_{K O H}^{\text {excess }}$ supports the hypothesis of cellulose charging up,

342 which claims that cellulose-ion interactions can turn cellulose into a

343 polyelectrolyte and cause its solvation. The cavity and interaction volumes of

344 cellulose, which are the dominant components of the partial molar volume of

345 cellulose, were in the order of $\mathrm{LiOH}<\mathrm{NaOH}<\mathrm{KOH}$, especially in the presence

346 of urea. It is believed that the alkali metal ion nearer to cellulose induces thermal

347 fluctuation of cellulose molecules more easily, and its hydrate causes more

348 electrostatic interactions and hydrogen bonding with cellulose. The calculation

349 results of $E^{u v}$ and $\Delta E^{R}$ indicate that urea strengthens not only the interaction

350 between cellulose and MOHs but also the solvent-solvent interaction, especially

$351 \mathrm{LiOH}$. The chemical potential of cellulose solvation was corrected by including

352 the advanced pressure correction and shows that the contribution of MOHs to

353 cellulose solvation was in the order $\mathrm{LiOH}>\mathrm{NaOH}>\mathrm{KOH}$, especially when urea

354 was present in the solvent. 3D-RISM-KH combined with KBI produced

355 quantitative information regarding the distribution and interaction of MOHs with

356 cellulose, clarified the role of $\mathrm{MOHs}$, especially $\mathrm{LiOH}$, in cellulose solvation, and

357 helped explain the preferential dissolution of cellulose in $\mathrm{LiOH} / \mathrm{urea}$ aqueous

358 solutions.

\section{Declarations}

360 Ethical approval

361 This article does not contain any studies with human participants or animals performed by

362 any of the authors.

364 Conflict of interest

365 The authors declare that they have no known competing financial interests or personal

366 relationships that could have appeared to influence the work reported in this paper.

\section{Acknowledgment}

369 This work was supported by Yonsei University and PaiChai University research grants in 2020. 


\section{References}

372 Ben-Naim A (1978) Standard thermodynamics of transfer. Uses and misuses J Phys Chem 82:792-

$373 \quad 803$

374 Bialik E, Stenqvist B, Fang Y, Östlund Å, Furo I, Lindman BR, Lund M, Bernin D (2016)

375 Ionization of cellobiose in aqueous alkali and the mechanism of cellulose dissolution J Phys Chem

376 Lett 7:5044-5048

377 Cai J, Zhang L (2005) Rapid dissolution of cellulose in LiOH/urea and $\mathrm{NaOH} /$ urea aqueous

378 solutions Macromol Biosci 5:539-548

379 Case D, Betz R, Cerutti D, Cheatham T, Darden T, Duke R AMBER16, 2016 San Francisco

380 Chalikian TV, Breslauer KJ (1996) On volume changes accompanying conformational transitions

381 of biopolymers Biopolymers 39:619-626

382 Chen CR, Makhatadze GI (2015) ProteinVolume: calculating molecular van der Waals and void

383 volumes in proteins BMC Bioinf 16:1-6

384 Gallicchio E, Kubo M, Levy RM (2000) Enthalpy- entropy and cavity decomposition of alkane

385 hydration free energies: Numerical results and implications for theories of hydrophobic solvation J

386 Phys Chem B 104:6271-6285

387 Giambaşu GM, Gebala MK, Panteva MT, Luchko T, Case DA, York DM (2015) Competitive

388 interaction of monovalent cations with DNA from 3D-RISM Nucleic Acids Res 43:8405-8415

389 Giambaşu GM, Luchko T, Herschlag D, York DM, Case DA (2014) Ion counting from explicit-

390 solvent simulations and 3D-RISM Biophys J 106:883-894

391 Gomes TC, Skaf MS (2012) Cellulose-Builder: A toolkit for building crystalline structures of

392 cellulose J Comput Chem 33:1338-1346

393 Gusarov S, Pujari BS, Kovalenko A (2012) Efficient treatment of solvation shells in 3D molecular

394 theory of solvation. J Comput Chem 33:1478-1494

395 Huh E, Yang J-H, Lee C-H, Ahn I-S, Mhin BJ (2020) Thermodynamic analysis of cellulose

396 complex in $\mathrm{NaOH}$-urea solution using reference interaction site model Cellulose 27:6767-6775

397 Imai T (2007a) Molecular theory of partial molar volume and its applications to biomolecular

398 systems. Condens Matter Phys 10:343-361

399 Imai T, Ohyama S, Kovalenko A, Hirata F (2007b) Theoretical study of the partial molar volume

400 change associated with the pressure-induced structural transition of ubiquitin. Protein Sci 16:

$401 \quad 1927-1933$

402 Jakalian A, Bush BL, Jack DB, Bayly CI (2000) Fast, efficient generation of high-quality atomic

403 charges. AM1-BCC model: I. Method. J Comput Chem 21:132-146

404 Jakalian A, Jack DB, Bayly CI (2002) Fast, efficient generation of high-quality atomic charges.

405 AM1-BCC model: II. Parameterization and validation. J Comput Chem 23: 1623-1641

406 Kovalenko A (2013) Multiscale modeling of solvation in chemical and biological nanosystems and

407 in nanoporous materials. Pure Appl Chem 85:159-199

408 Kovalenko A (2017) Multiscale modeling of solvation. In: Breitkopf C, Swider-Lyons K (eds)

409 Springer handbook of electrochemical energy. Springer, Heidelberg, pp 95-139 
410 Kovalenko A, Gusarov S (2018) Multiscale methods framework: self-consistent coupling of

411 molecular theory of solvation with quantum chemistry, molecular simulations, and dissipative

412 particle dynamics. Phys Chem Chem Phys 20:2947-2969

413 Krüger P, Schnell SK, Bedeaux D, Kjelstrup S, Vlugt TJ, Simon J-M (2013) Kirkwood-Buff

414 integrals for finite volumes. J Phys Chem Lett 4:235-238

415 Lazaridis T (2000) Solvent reorganization energy and entropy in hydrophobic hydration. J Phys

416 Chem B 104:4964-4979

417 Lee B (1983) Partial molar volume from the hard-sphere mixture model. J Phys Chem 87:112-118

418 Misin M (2017) Can approximate integral equation theories accurately predict solvation

419 thermodynamics? Ph.D. thesis, University of Strathclyde. arXiv:1704.05246.

420 Misin M, Vainikka PA, Fedorov MV, Palmer DS (2016) Salting-out effects by pressure-corrected

421 3D-RISM. J Chem Phys 145:194501

422 Nicol TW, Isobe N, Clark JH, Shimizu S (2017) Statistical thermodynamics unveils the dissolution

423 mechanism of cellobiose Phys Chem Chem Phys 19:23106-23112

424 Patel N, Dubins DN, Pomes R, Chalikian TV (2011) Parsing partial molar volumes of small

425 molecules: A molecular dynamics study. J Chem Phys B 115:4856-4862

426 Sergiievskyi VP, Jeanmairet G, Levesque M, Borgis D (2014) Fast computation of solvation free

427 energies with molecular density functional theory: Thermodynamic-ensemble partial molar

428 volume corrections. J Phys Chem Lett 5:1935-1942

429 Shimizu S (2004) Estimating hydration changes upon biomolecular reactions from osmotic stress,

430 high pressure, and preferential hydration experiments. Proc Natl Acad Sci U S A 101:1195-1199

431 Shimizu S, Booth JJ, Abbott S (2013) Hydrotropy: binding models vs. statistical thermodynamics.

432 Phys Chem Chem Phys 15:20625-20632

433 Wang S, Lyu K, Sun P, Lu A, Liu M, Zhuang L, Zhang L (2017) Influence of cation on the

434 cellulose dissolution investigated by MD simulation and experiments. Cellulose 24:4641-4651

435 Wernersson E, Stenqvist B, Lund M (2015) The mechanism of cellulose solubilization by urea

436 studied by molecular simulation. Cellulose 22:991-1001

437 Xiong B, Zhao P, Cai P, Zhang L, Hu K, Cheng G (2013) NMR spectroscopic studies on the

438 mechanism of cellulose dissolution in alkali solutions. Cellulose 20:613-621 
Figures

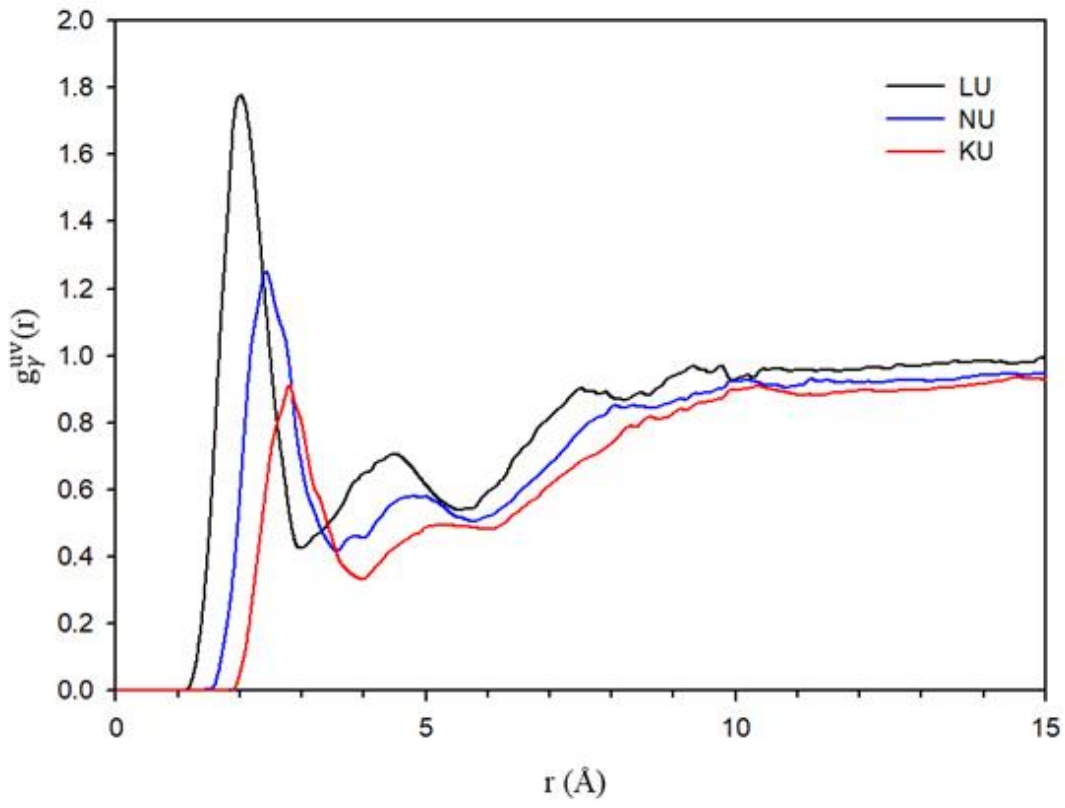

440

441 Fig. 1 Pair distribution function $\left(g_{\gamma}^{u v}(r)\right)$ of $\mathrm{Li}^{+}, \mathrm{Na}^{+}$and $\mathrm{K}^{+}$to the $\mathrm{O} 2$ atoms of cellulose in 442 solvents LU, NU, and KU. Refer to Fig. 2 of Huh et al. (Huh et al. 2020) for the O2 atoms of 443 cellulose. 


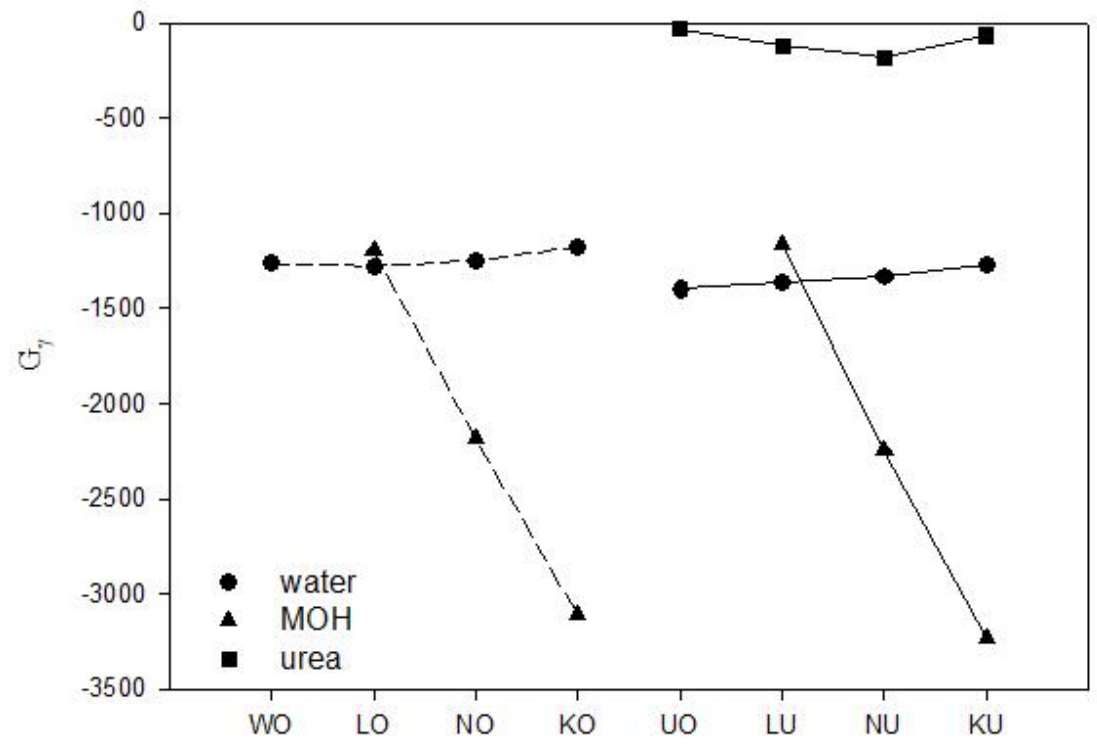

444

445 Fig. 2 Kirkwood Buff Integral $\left(G_{\gamma}\right)$ for a solvent species $\gamma$ in 8 different solvents: $(O)$ for water;

446 ( $\mathbf{\Delta}$ ) for an alkali metal hydroxide (MOH); and ( $\mathbf{\square})$ for urea. The unit of $G_{\gamma}$ is $\AA^{3}$ per cellulose 447 molecule.

448 
(a)

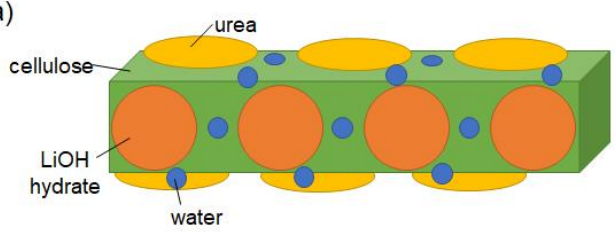

(b)

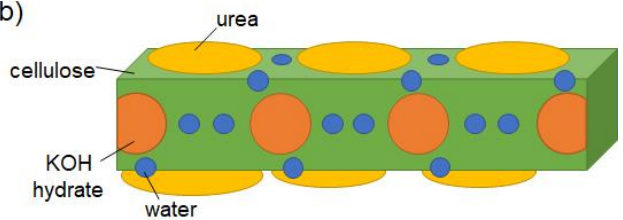

450 Fig. 3 Schematic depiction of solvent species around the single cellulose molecule in solvents LU

451 (A) and KU (B). Urea (yellow circle) and hydrated $\mathrm{MOH}$ (orange colored circle) are positioned near 452 the hydrophobic and hydrophilic parts of cellulose, respectively. Note the differences in the numbers 453 of $\mathrm{MOH}$ and water molecules (blue circle) between (A) and (B). 


\section{Table}

455 Table 1. Excess number of solvent species $\gamma\left(N_{\gamma}^{\text {excess }}\right)$ in various solvents.

\begin{tabular}{cccc}
\hline solvent & Water & $\mathrm{LiOH} / \mathrm{NaOH} / \mathrm{KOH}$ & Urea \\
\hline $\mathrm{LU}$ & -0.8 & 0.0 & 1.4 \\
$\mathrm{NU}$ & 0.4 & -1.2 & 1.3 \\
$\mathrm{KU}$ & 2.4 & -2.3 & 1.5 \\
$\mathrm{UO}$ & -1.2 & & 1.5
\end{tabular}

456 
457 Table 2. Cavity volume $\left(V_{C}\right)$, interaction volume $\left(V_{I}\right)$, ideal fluctuation volume $\left(V_{i d}\right)$, and partial 458 molar volume $(\bar{V})$ of cellulose in solvents $\mathrm{LU}, \mathrm{NU}$, and $\mathrm{KU}^{\mathrm{a}}$

\begin{tabular}{cccccc}
\hline solvent & $V_{C}$ & $V_{I}$ & $V_{i d}$ & $\bar{V}$ & $V_{T}{ }^{\mathrm{b}}$ \\
\hline $\mathrm{LU}$ & 1335.0 & -57.5 & 1.5 & 1278.8 & 161.7 \\
$\mathrm{NU}$ & 1339.4 & -57.4 & 1.4 & 1283.4 & 166.1 \\
$\mathrm{KU}$ & 1347.1 & -50.5 & 1.3 & 1297.7 & 173.8 \\
$\mathrm{UO}^{\mathrm{c}}$ & 1360.5 & -48.7 & 1.8 & 1313.6 & 187.2 \\
\hline
\end{tabular}

$459{ }^{\mathrm{a}} \bar{V}=V_{C}+V_{I}+V_{i d}$. The unit is $\AA^{3}$ per cellulose molecule.

$460{ }^{\mathrm{b}} V_{T}$ is the thermal volume and calculated from $V_{T}=V_{C}-V_{M}$ where $V_{M}=1173.3 \AA^{3}$.

$461{ }^{\mathrm{c}}$ Data in solvent UO are given for comparison.

462 
463 Table 3. $V_{C, \gamma}$ and $V_{I, \gamma}$, components of $V_{C}$ and $V_{I}$ for solvent species $\gamma^{\text {a }}$

\begin{tabular}{ccccccc}
\hline solvent & $V_{C, \text { water }}$ & $V_{C, \mathrm{MOH}}$ & $V_{C, \text { urea }}$ & $V_{I, \text { water }}$ & $V_{I, \text { MOH }}$ & $V_{I, \text { urea }}$ \\
\hline LU & 1234.7 & 16.4 & 84.0 & -50.9 & -3.5 & -3.4 \\
$\mathrm{NU}$ & 1210.1 & 45.0 & 84.3 & -49.9 & -4.0 & -3.4 \\
$\mathrm{KU}$ & 1186.6 & 75.1 & 85.4 & -43.4 & -4.2 & -3.0 \\
$\mathrm{UO}^{\mathrm{b}}$ & 1277.7 & & 82.8 & -45.9 & & -2.8 \\
\hline
\end{tabular}

464 a The unit is $\AA^{3}$ per cellulose molecule. Values of $V_{C}$ and $V_{I}$ are shown in Table 2.

$465{ }^{\mathrm{b}}$ Data in solvent UO are given for comparison.

466 
467 Table 4. Solvation energy and solvation entropy of cellulose in solvents LU, NU, and KU at 261

$468 \quad \mathrm{~K}^{\mathrm{a}}$

\begin{tabular}{ccrrrr}
\hline solvent & \multicolumn{1}{c}{$\Delta E$} & \multicolumn{1}{c}{$E^{u v}$} & $\Delta E^{R}\left(\approx T \Delta S^{R}\right)$ & \multicolumn{1}{c}{$T \Delta S$} & \multicolumn{1}{c}{$T \Delta S^{u v}$} \\
\hline LU & -1672.4 & -481.4 & -1191.0 & -1709.8 & -518.7 \\
& & $(-411.8)[-49.7]$ & $(-1086.0)[-101.2]$ & & $(-460.6)[-29.3]$ \\
NU & -1600.9 & -470.9 & -1130.0 & -1658.8 & -528.8 \\
& & $(-415.0)[-36.1]$ & $(-1067.3)[-61.7]$ & $(-471.2)[-27.9]$ \\
KU & -1537.9 & -450.7 & -1087.2 & -1631.5 & -544.3 \\
& & $(-403.9)[-26.9]$ & $(-1054.7)[-33.2]$ & & $(-482.7)[-30.2]$ \\
UO ${ }^{\text {b }}$ & -142.2 & -306.6 & 164.3 & -231.9 & -67.6 \\
& & $(-287.4)$ & $(159.7)$ & $(-51.0)$ \\
\hline
\end{tabular}

$469{ }^{a} \Delta E=E^{u v}+\Delta E^{R}$ and $T \Delta S=T \Delta S^{u v}+T \Delta S^{R}$. The superscripts uv and R denote solute-solvent

470 interaction and solvent reorganization (solvent-solvent interaction), respectively. Numbers in

471 round and square brackets indicate the contributions of water and $\mathrm{MOHs}$, respectively. The unit of 472 energy is kcal per mole of cellulose.

$473{ }^{\mathrm{b}}$ Data in solvent UO are given for comparison.

474 
475 Table 5. Effect of MOHs and urea on the direct solvation energy (uv) and the solvent 476 reorganization energy $(\mathrm{R})^{\mathrm{a}}$

\begin{tabular}{ccrrc}
\hline $\mathrm{MOH}$ & $\Delta E_{\mathrm{MOH}-W O}^{u v}$ & \multicolumn{1}{c}{$\Delta E_{M O H-U O}^{u v}$} & $\Delta \Delta E_{M O H-W O}^{R}$ & $\Delta \Delta E_{M O H-U O}^{R}$ \\
\hline $\mathrm{LiOH}$ & -17.2 & -174.8 & -3.8 & -1355.3 \\
$\mathrm{NaOH}$ & -4.9 & -164.3 & 16.5 & -1294.3 \\
$\mathrm{KOH}$ & -3.7 & -144.1 & 28.2 & -1251.5 \\
\hline
\end{tabular}

477 a These energies are calculated at $261 \mathrm{~K}$ and their unit is kcal per mole of cellulose. The definitions 478 of $\Delta E_{\mathrm{MOH}-W O}^{u v}, \Delta E_{\mathrm{MOH}-U O}^{u v}, \Delta \Delta E_{\mathrm{MOH}-\mathrm{WO}}^{R}$, and $\Delta \Delta E_{\mathrm{MOH}-U \mathrm{OO}}^{R}$ are shown in Eqs. (8)-(11). 
480 Table 6. Chemical potentials of cellulose solvation without $(\Delta \mu)$ and with $\left(\Delta \mu_{P C+}\right)$, the advanced 481 pressure correction $(\mathrm{PC}+)$ in solvents $\mathrm{LU}, \mathrm{NU}$, and $\mathrm{KU}$ at $261 \mathrm{~K}^{\mathrm{a}}$

\begin{tabular}{ccc}
$\mathrm{MOH}$ & $\Delta \mu$ & $\Delta \mu_{P C+}$ \\
\hline $\mathrm{LU}$ & 37.4 & -193.8 \\
$\mathrm{NU}$ & 57.9 & -188.6 \\
$\mathrm{KU}$ & 93.7 & -178.4 \\
$\mathrm{UO}^{\mathrm{b}}$ & 89.7 & -107.9
\end{tabular}

$482{ }^{a}$ The unit of energy is kcal per mole of cellulose.

$483{ }^{\mathrm{b}}$ Data in solvent UO are given for comparison. 
Figures

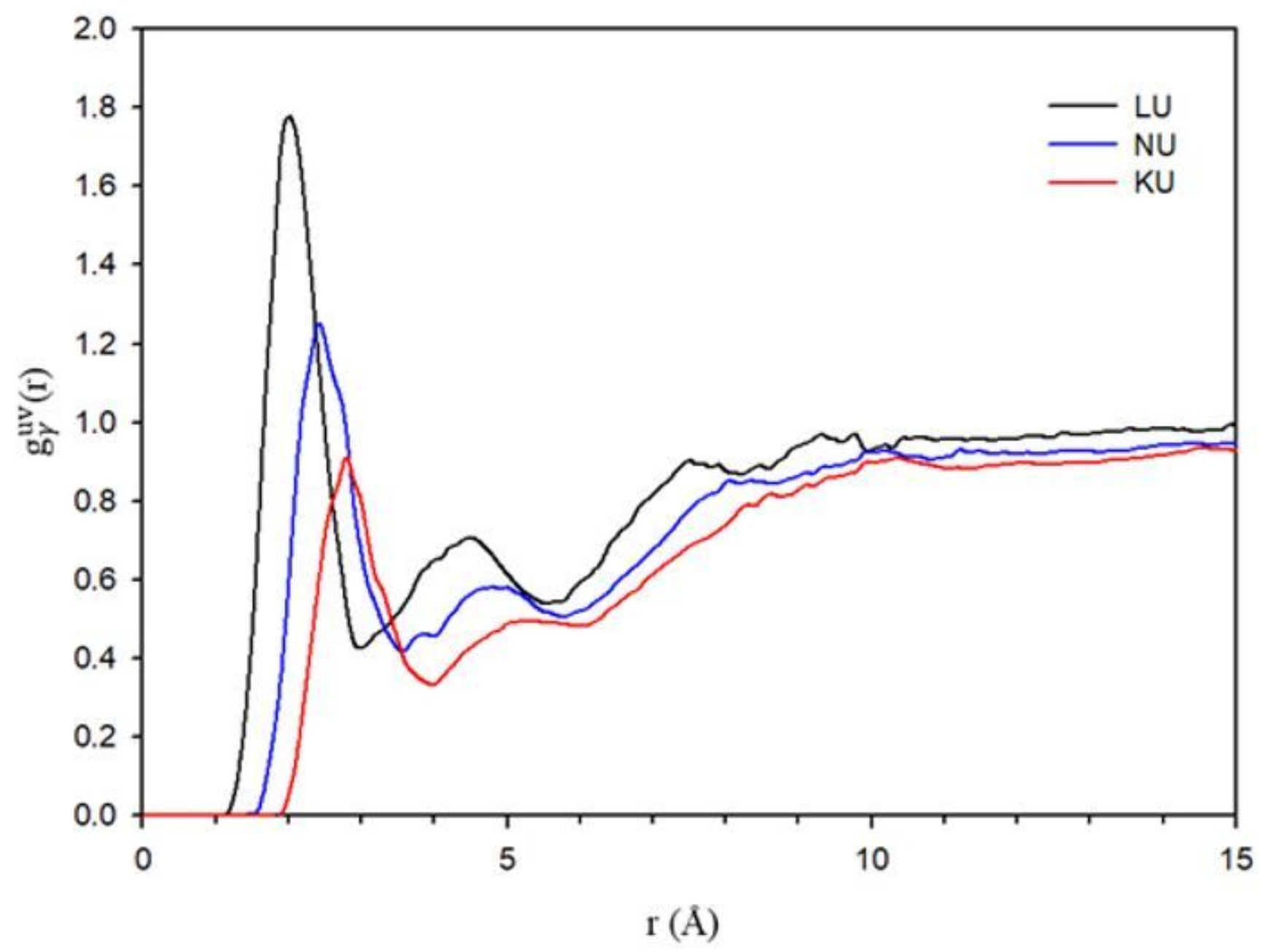

Figure 1

Pair distribution function ( $\mathrm{g}_{-} \mathrm{Y}^{\wedge} \mathrm{uv}(\mathrm{r})$ ) of $\mathrm{Li}+, \mathrm{Na}+$ and $\mathrm{K}+$ to the $\mathrm{O} 2$ atoms of cellulose in solvents $\mathrm{LU}, \mathrm{NU}$, and KU. Refer to Fig. 2 of Huh et al. (Huh et al. 2020) for the 02 atoms of cellulose. 


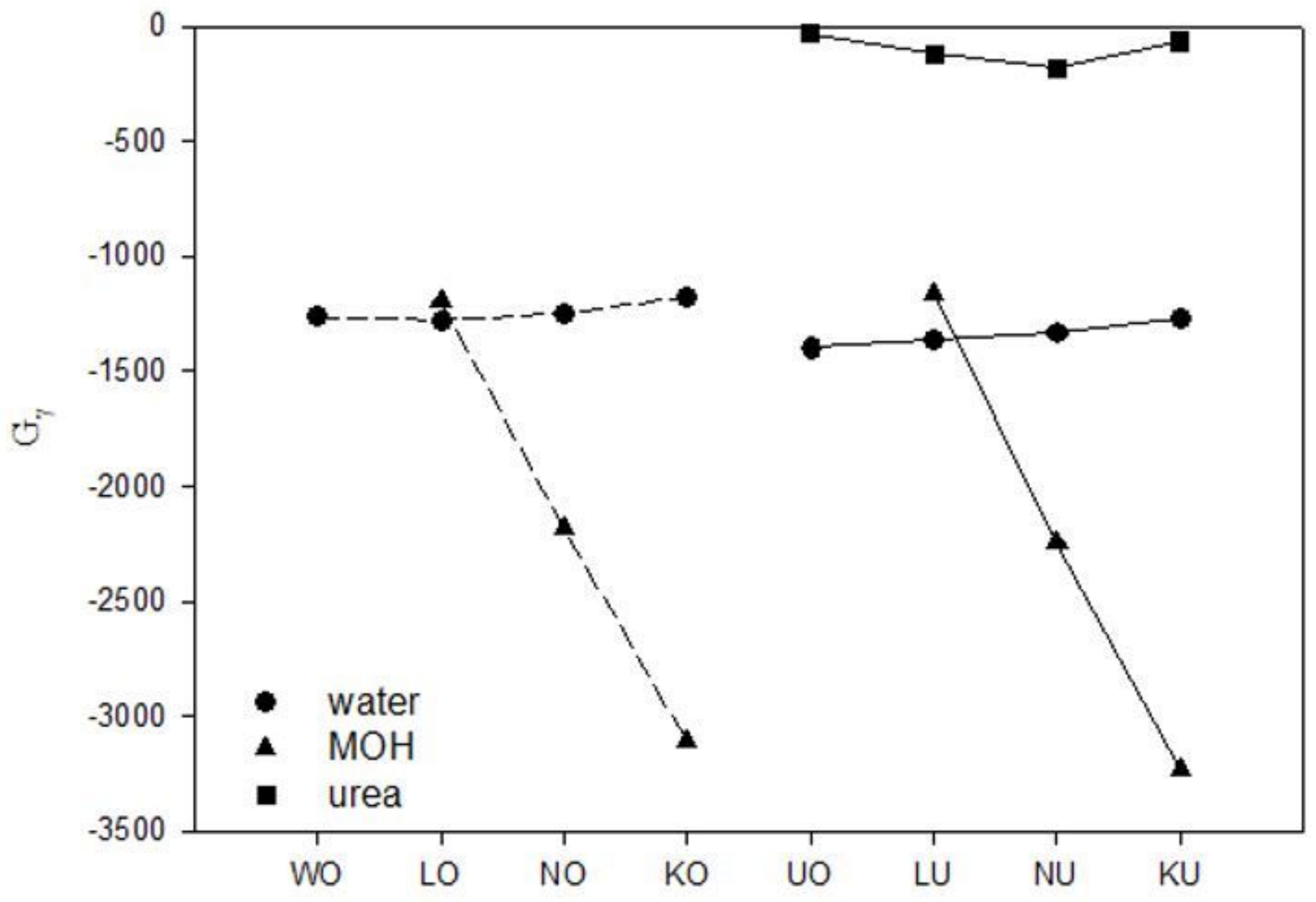

Figure 2

Kirkwood Buff Integral (G_Y) for a solvent species $y$ in 8 different solvents: ( () for water; ( () for an alkali

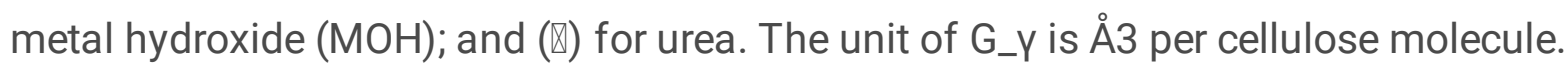

(a)

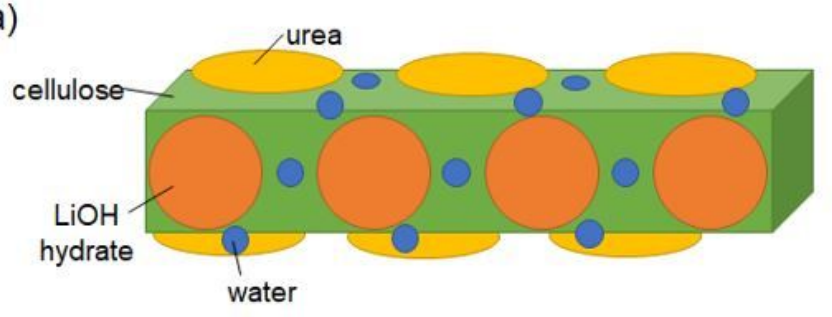

(b)

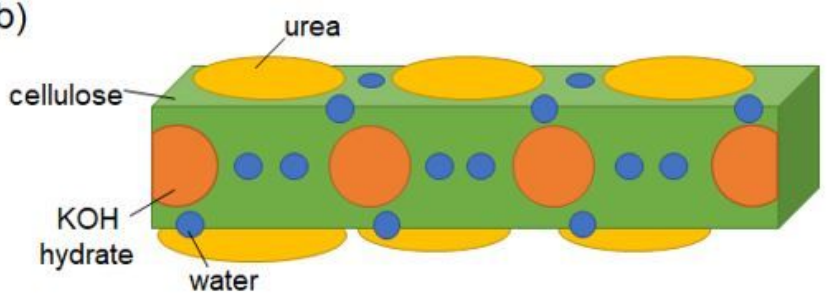

\section{Figure 3}

Schematic depiction of solvent species around the single cellulose molecule in solvents $L U(A)$ and KU (B). Urea (yellow circle) and hydrated $\mathrm{MOH}$ (orange colored circle) are positioned near the hydrophobic 
and hydrophilic parts of cellulose, respectively. Note the differences in the numbers of $\mathrm{MOH}$ and water molecules (blue circle) between (A) and (B).

\section{Supplementary Files}

This is a list of supplementary files associated with this preprint. Click to download.

- SupplementaryInformation.docx 